\title{
Antero- und retrograde elastisch-stabile Markraumschienung (ESIN) bei Humerusfrakturen im Kindesalter
}

\author{
Theddy F. Slongo ${ }^{1}$
}

\section{Zusammenfassung \\ Operationsziel}

Sichere und definitive Stabilisierung stark verschobener, instabiler sowie mit konservativen Methoden nicht retinierbarer Humerusfrakturen, subkapital, im Bereich des Schafts und suprakondylär.

\section{Indikationen}

Richten sich am Humerus nach zwei Gesichtspunkten: nach Morphologie (stark verschobene Querfrakturen, lange Schräg- und Spiralbrüche sowie komplexe Brüche) und Stabilität (völlig instabile Frakturen) sowie heute nach sozioökonomischen Gründen und Komfort. Daher kennt man absolute und relative Indikationen.

\section{Kontraindikationen}

Stabile, unverschobene Brüche.

\section{Operationstechnik}

Richtet sich nach den Prinzipien der ESIN-Technik (elastisch-stabile Markraumschienung). Klare Darstellung der
Eintrittspunkte auf der lateralen Seite; Eröffnung des Markraums mit dem Pfriem oder Bohrer; Einbringen der beiden Nägel, wobei ein Nagel C-förmig, der andere $\mathrm{S}$-förmig vorgebogen ist.

Weiterbehandlung

Gipsverbandfreie, funktionelle Weiterbehandlung; je nach Angst und Schmerz kann eine Schlinge verwendet werden.

\section{Ergebnisse}

Bei korrekter Indikation und Technik ist am Humerus immer ein perfektes Ergebnis zu fordern; Fehler liegen immer beim Chirurgen!

\section{Schlüsselwörter}

Humerus - Subkapitale Humerusfraktur . Suprakondyläre Fraktur · ESIN · TEN · Elastischstabile intramedulläre Nagelung · Frakturklassifikation
Oper Orthop Traumatol 2008;20:373-86

DOI 10.1007/s00064-008-1409-5

${ }^{1}$ Abteilung für Pädiatrische Chirurgie, Universitätskinderklinik, Inselspital, Bern, Schweiz. 


\section{Ante- and Retrograde Intramedullary Nailing of Humerus Fractures}

\section{Abstract \\ Objective}

Safe and definitive stabilization of fully displaced, unstable fractures of the humerus which cannot be fixed conservatively; plaster cast: subcapital humerus fractures, fractures of the shaft, and supracondylar fractures.

\section{Indications}

These depend on two major factors: first, on the morphology (strongly displaced transverse fractures, long oblique and spiral fractures, and comminuted fractures) and the stability of the fracture, and second, more often on socioeconomic aspects and comfort. Therefore, absolute and relative indications are known.

\section{Contraindications}

Stable and undisplaced fractures.

\section{Surgical Technique}

Follows the rules and principles of the ESIN technique (elastic stable intramedullary nailing). Preparation of the lateral/ radial entry points; opening of the medullary canal with an awl or drill; introduction of both precontoured nails, of which one is C-shaped, and the other S-shaped.

\section{Postoperative Management}

No plaster cast or splint necessary, always functional postoperative treatment; exceptions are pain or anxiety of the child or parents (arm sling).

\section{Results}

In case of correct indication and operative technique, an optimal result is to be expected; the responsibility for possible failures lies always on the surgeon!

\section{Key Words \\ Humerus · Subcapital humerus fracture $\cdot$ Supra- condylar humerus fracture · ESIN · Elastic stable intramedullary nailing · Fracture classification}

\section{Vorbemerkungen}

Frakturen des proximalen oder distalen meta-epiphysären Humerus werden auch heute noch häufig, am distalen Ende praktisch ausschließlich mit Kirschner-Drähten stabilisiert. Bei der suprakondylären Humerusfraktur werden die mit diesem Verfahren verbundenen Einschränkungen von dem betroffenen Kind gut toleriert. Dagegen bereitet die Fixation einer Humerusschaftfraktur mit einem Fixateur externe sowie einer proximalen Fraktur mit transkutanen Kirschner-Drähten doch erhebliche Probleme. Der Nachteil einer zweiten Narkose zur Implantatentfernung bei der elas-

\section{Operationsprinzip und -ziel}

Sichere und definitive Stabilisierung dislozierter und instabiler Humerusfrakturen mit minimalinvasiv eingebrachten elastischen Nägeln (ESIN). Bei Schaftfrakturen sowie subkapitalen Frakturen werden die Nägel von distal und radial, ausnahmsweise distal, bilateral und radioulnar eingebracht. Bei distalen und suprakondylären Frakturen wird die sog. anterograde Technik von proximal angewandt.

Radial wie auch in Ausnahmefällen ulnar wird der Humerus distal dargestellt. Bei der anterograden Technik tisch-stabilen intramedullären Nagelung (ESIN) kompensiert laut Aussagen der Kinder und Eltern die Nachteile anderer Verfahren bei Weitem.

Die Beschreibung der Indikationen stützt sich in diesem Beitrag auf die neue Comprehensive AO Paediatric Long Bone Classification [8].

Die Verwendung der ESIN zur Stabilisierung von Humerusfrakturen ist nicht neu und wurde bereits von Metaizeau beschrieben [5]. Ausführlich wird diese Behandlungsmethode ebenfalls im AO Manual of Fracture Management. Elastic Stable Intramedullary Nailing (ESIN) in Children dargestellt [1].

wird die Kortikalis über einen minimalen Zugang perforiert.

Bei vorbestehendem Schaden des Nervus radialis sollte bei dieser Technik der Nerv über einen kleinen, direkten Zugang visualisiert werden, da er bei Lage innerhalb des Frakturspalts durch den Nagel weiter beschädigt oder vollends zerstört werden kann. Dieses Vorgehen steht im Gegensatz zu dem bei konservativer Therapie, wo eine Symptomatik des Nervus radialis keine primäre Indikation zur Revision darstellt. 


\section{Vorteile}

- Biomechanisch zuverlässige und stabile Osteosynthese.

- Einfach erlernbar.

- Funktionelle bis teilbelastbare Nachbehandlung ohne zusätzlich Ruhigstellung.

- Teilstationäre Behandlung.

- Einwandfreie funktionelle und ästhetische Heilung zu erwarten.

- Keine irritierenden transkutanen Pins oder Kirschner-Drähte.

- Keine Pflege von Drähten oder Pins durch die Eltern notwendig.

- Prinzipiell keine funktionellen Einschränkungen während der Heilung.

- Problemloser Schulbesuch möglich.

\section{Nachteile}

- Zweite Narkose zur Implantatentfernung notwendig.

- Bei zu hoch oder falsch gewähltem Eintrittspunkt distal Gefahr der Schädigung des Nervus radialis (lateral) oder Nervus ulnaris (medial).

- Höhere Implantatkosten, die sich durch weniger häufige Nachkontrollen oder allfällige Nachrepositionen vermindern.

\section{Indikationen}

- Distale, suprakondyläre Humerusfrakturen:

- Unabhängig vom Alter alle instabilen und verschobenen Brüche (13 - M/3.1 III, 13 - M/3.1 IV, 13 M/3.2 III und $13-\mathrm{M} / 3.2$ IV, s. Abbildung 1).

- Humerusschaftfrakturen:

- Generell bei Kindern > 10 Jahre. Das Alter spielt eine eher untergeordnete Rolle, wobei die Grenze zum aktiven Vorgehen je nach Entwicklung des Kindes bei 10 Jahren liegen dürfte.

- Völlig instabile Frakturen sowie Mehrfragmentfrakturen mit Achsabweichung $>15^{\circ}$ oder Verkürzung (12 - D/4.1, 12 - D/4.2, 12 - D/5.1, 12 - D/5.2). Glücklicherweise verursachen Fehlstellungen am Humerus kaum funktionelle Probleme. Die Flexibilität der Schulter kompensiert auch schwerwiegendere Rotationsfehler. Trotzdem führen Achsabweichungen $>10-15^{\circ}$, vor allem auch bei Mädchen, $\mathrm{zu}$ ästhetisch schlechten Resultaten, so dass wir die Indikation zur chirurgischen Stabilisierung großzügig stellen.

- Kinder mit Polytrauma.

- Ipsilaterale Frakturen von Humerus und Unterarm.

- Begleitfrakturen an der unteren Extremität, wenn Gehkrücken benötigt werden.

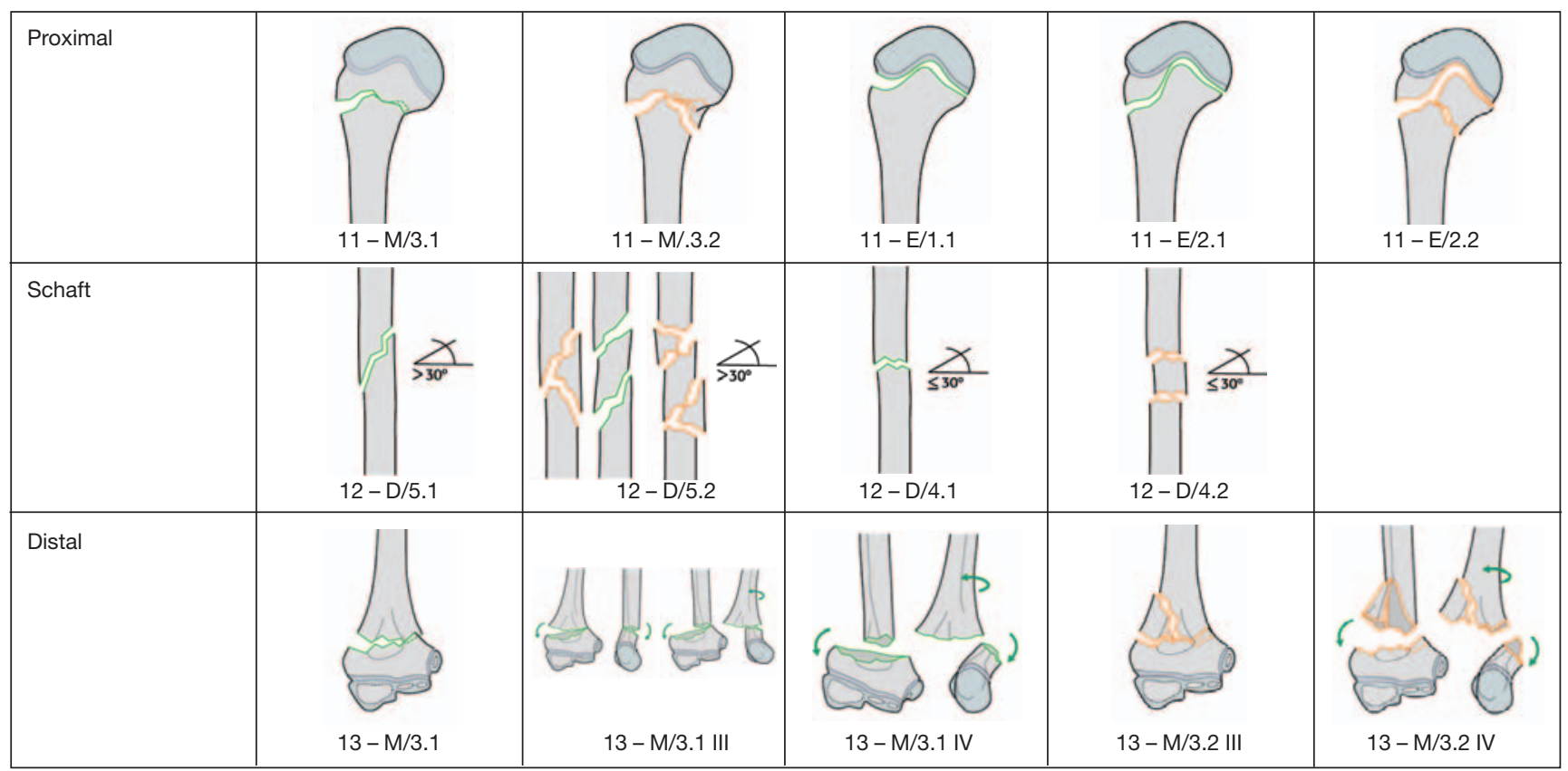

Abbildung 1

Zusammenstellung der Frakturtypen bezüglich Indikationen (aus [8]). 


\section{Abbildungen $2 \mathrm{a}$ bis $\mathbf{2 h}$}

Instrumente für die ESIN (Fa. Synthes; aus [1]).

a) Pfriem.

b) Handgriff.

c) Abgeschrägter Einschlagbolzen.

d) Schneidinstrument.

e) Aufschraubbare Verlängerungsstange.

f) Extraktionszange.

g) F-Hebel.

h) Endkappe.

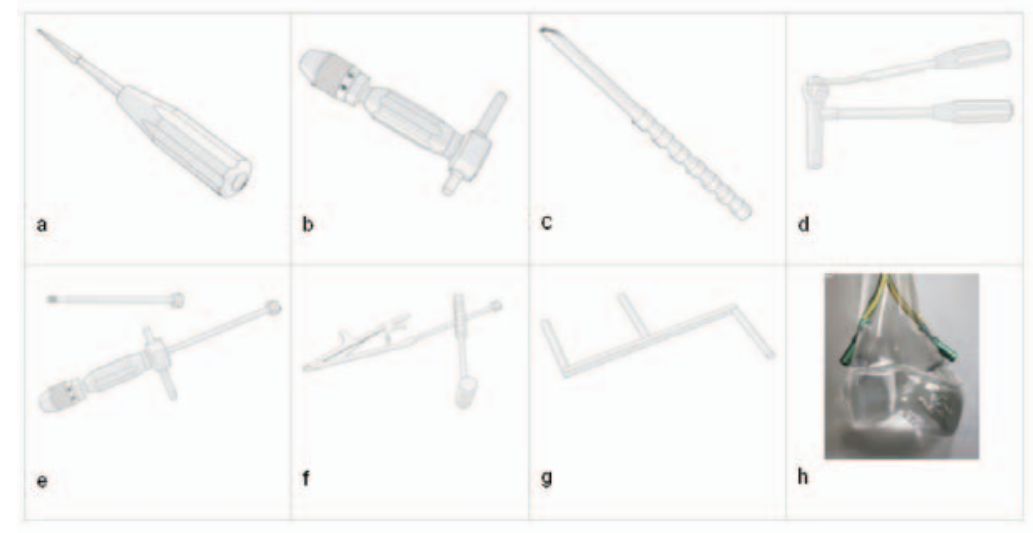

- Offene Frakturen vom Typ II und III nach Gustilo.

- Pathologische Frakturen oder generalisierte Skeletterkrankungen, die eine konservative Therapie unmöglich machen.

- Subkapitale Humerusfrakturen:

- Das Alter ist hier nicht der primär limitierende Faktor für ein chirurgisches Vorgehen bei völlig instabilen und verschobenen Brüchen.

- Alle instabilen und verschobenen Brüche bei Kindern $<10$ Jahre und Achsabweichungen $>30^{\circ} \mathrm{Va}-$ rus, Ante- oder Rekurvation oder $>10^{\circ}$ Valgus.

- Bei Kindern $>10$ Jahre Achsabweichung $>10^{\circ}$ sowie Verkürzungen (Frakturtypen: 11 - M/3.1, 11 $\mathrm{M} / 3.2,11-\mathrm{E} / 1.1$ und $11-\mathrm{E} / 2.1)$.

\section{Kontraindikationen}

- Keine.

\section{Patientenaufklärung}

- Vor- und Nachteile eines konservativen und operativen Vorgehens müssen mit Eltern und Kind besprochen werden, da, wie oben erwähnt, Fehlverheilungen und Heilungsstörungen praktisch keine funktionellen, sondern höchstens ästhetische Probleme bieten.

- Geringes Infektionsrisiko, nicht höher als bei perkutanen Kirschner-Drähten.

- Hautirritation an den Nagelenden mit gelegentlichen Schmerzen, die nach Implantatentfernung immer abklingen.

- Darstellung des Nervus ulnaris bei ulnarem Vorgehen.

- Verletzung des Nervus radialis bei zu hoher oder zu tiefer Eintrittsstelle bei monolateraler, radialer Technik.

- Keine Physiotherapie nötig.

- Partieller Gebrauch des Arms möglich.
- Sportverbot für ca. 5-6 Wochen.

- Zweite Narkose zur Implantatentfernung.

- Kleine Narben sichtbar.

- Falls keine geschlossene Reposition möglich, kleiner Hautschnitt über Fraktur notwendig.

- Schädigung des Nervus radialis mit der Nagelspitze.

\section{Operationsvorbereitungen}

- Keine speziellen Vorbereitungen erforderlich.

- Falls keine Schädigung von Gefäßen oder Nerven vorliegt, kann die Operation auch am Folgetag durchgeführt werden [1-3].

\section{Instrumentarium und Implantate}

- Wir verwenden das Orginalinstrumentarium der Firma Synthes mit den dazugehörigen Titan- oder Stahlimplantaten (Abbildungen 2a bis 2h). Damit lassen sich die Durchleuchtungszeiten verringern, und es bietet Hilfe bei der Implantation der Nägel, der indirekten Reposition sowie beim Kürzen der Nägel.

- Standardinstrumente:

- Pfriem (Abbildung 2a).

- Markierter Handgriff mit stabilem Knauf zum Einschlagen (Abbildung 2b).

- Abgeschrägter Einschlagbolzen mit Loch und definiertem Überstand (Abbildung 2c).

- Bolzenschneider für alle Nageldurchmesser (Abbildung 2d).

- Aufschraubbare Verlängerungsstange, verwendbar für Handgriff und Extraktionszange (Abbildung 2e).

- Extraktionszange (Abbildung 2f).

- Optionale Zusatzinstrumente:

-F-Hebel zur indirekten Reposition (Abbildung $2 \mathrm{~g})$.

- Verriegelbare Endkappen zur Erhöhung der axialen Stabilität (Abbildung 2h). 


\section{Anästhesie und Lagerung}

- Vorzugsweise Intubationsnarkose, Regionalanästhesien bei Kindern wenig geeignet.

- Bei nüchternem Kind Verwendung einer Larynxmaske.

- Rückenlage.

- Operationstisch umgedreht, Schulter und Kopf auf dem frei überstehenden Teil (Abbildung 3).

- Kind ganz zur Gegenseite gelagert, damit die betroffenen Körperteile frei durchleuchtbar sind und nicht durch die Tischumrandung verdeckt werden.

- Fixierung des Oberkörpers zur Gegenseite mit Tuch.

- Die Durchleuchtungszeit sollte so gering wie möglich gehalten werden, daher Bildverstärker auf Impulsbestrahlung stellen (,schnelles Auge“).

- Nur einzelne kurze Bildsequenzen auslösen, den sog. Bleifuß vermeiden!

\section{Operationstechnik}

\section{Abbildungen 4 bis 14}

Für die Bestimmung der Nageldicke wird anhand des ausgedruckten Röntgenbilds oder auf elektronischem Weg an einer PACS-Konsole die Weite des Markkanals im Isthmusbereich ausgemessen. Die Nageldicke beträgt $30-40 \%$ dieses Durchmessers.

\section{Abbildungen 4a bis $4 c$}

Prinzipiell unterscheiden wir drei verschiedene ESIN-Techniken am Humerus. Diese müssen frakturspezifisch, d.h. aufgrund der Frakturmorphologie einerseits und der Lokalisation andererseits, gewählt werden. Werden diese Prinzipien nicht beachtet, kann die Methode scheitern!

Folgende drei Methoden kommen zur Anwendung [1]:

a) Retrograde distale, radiale, monolaterale Technik für mittleres Schaftdrittel und proximale/subkapitale Humerusfrakturen.

b) Retrograde distale, bilaterale, radioulnare Technik für komplexere Schaftfrakturen.

c) Anterograde proximale/radiale Technik für distales Humerusdrittel und suprakondyläre Frakturen.

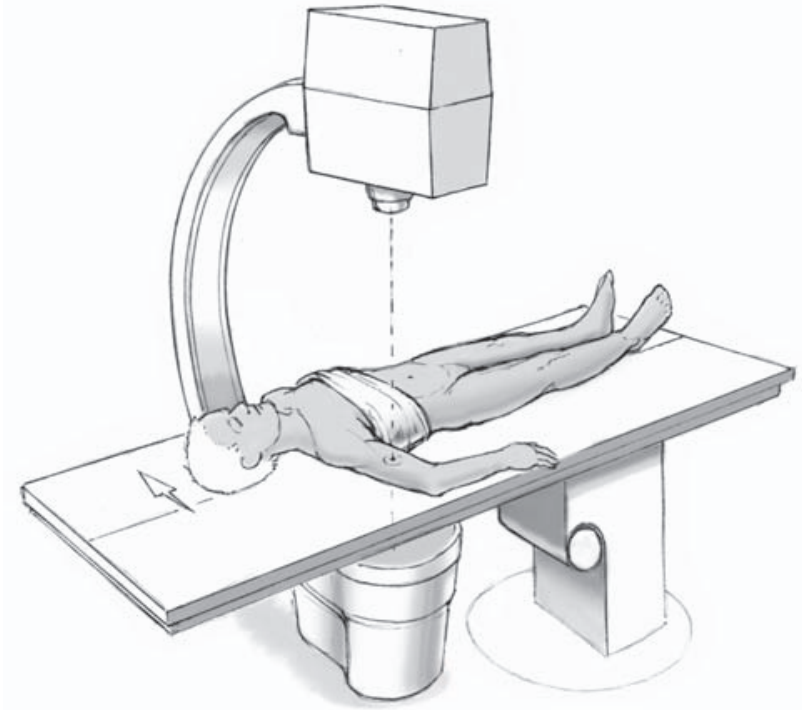

Abbildung 3

Lagerung auf dem ausgefahrenen oder umgedrehten Operationstisch. Patient zur Gegenseite gelagert, was eine freie Sicht mit Bildverstärker über den ganze Oberarm ermöglicht.
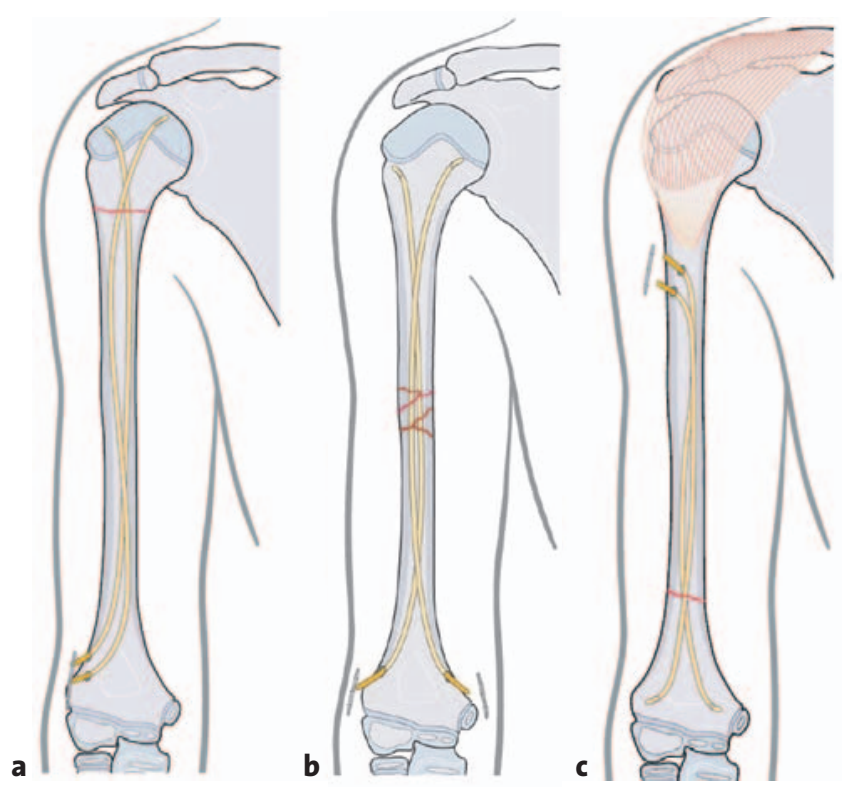


\section{Retrograde radiale, einseitige Technik am Beispiel einer subkapitalen Humerusfraktur}

\section{Abbildungen 5 a und $\mathbf{5 b}$}

Hautinzision und Zugang zum Humerus.

Hautinzision radial über eine Länge von ca. 4-5 cm und ca. $1 \mathrm{~cm}$ proximal des lateralen Kondylus (a). Die Eintrittspunkte sollen gegeneinander versetzt liegen, um ein Aufsplittern des Knochens zu verhindern. Man beachte den Verlauf des Nervus radialis. Es empfiehlt sich, einen kleinen Hohmann-Haken zu platzieren, der nicht bis zur Gegenseite reichen muss. Ablösen der Muskulatur und des Periosts am Septum intermusculare auf einer Länge von $4 \mathrm{~cm}$ (b). Zugang zum Humerus durch direktes Eingehen auf die laterale Crista humeri.
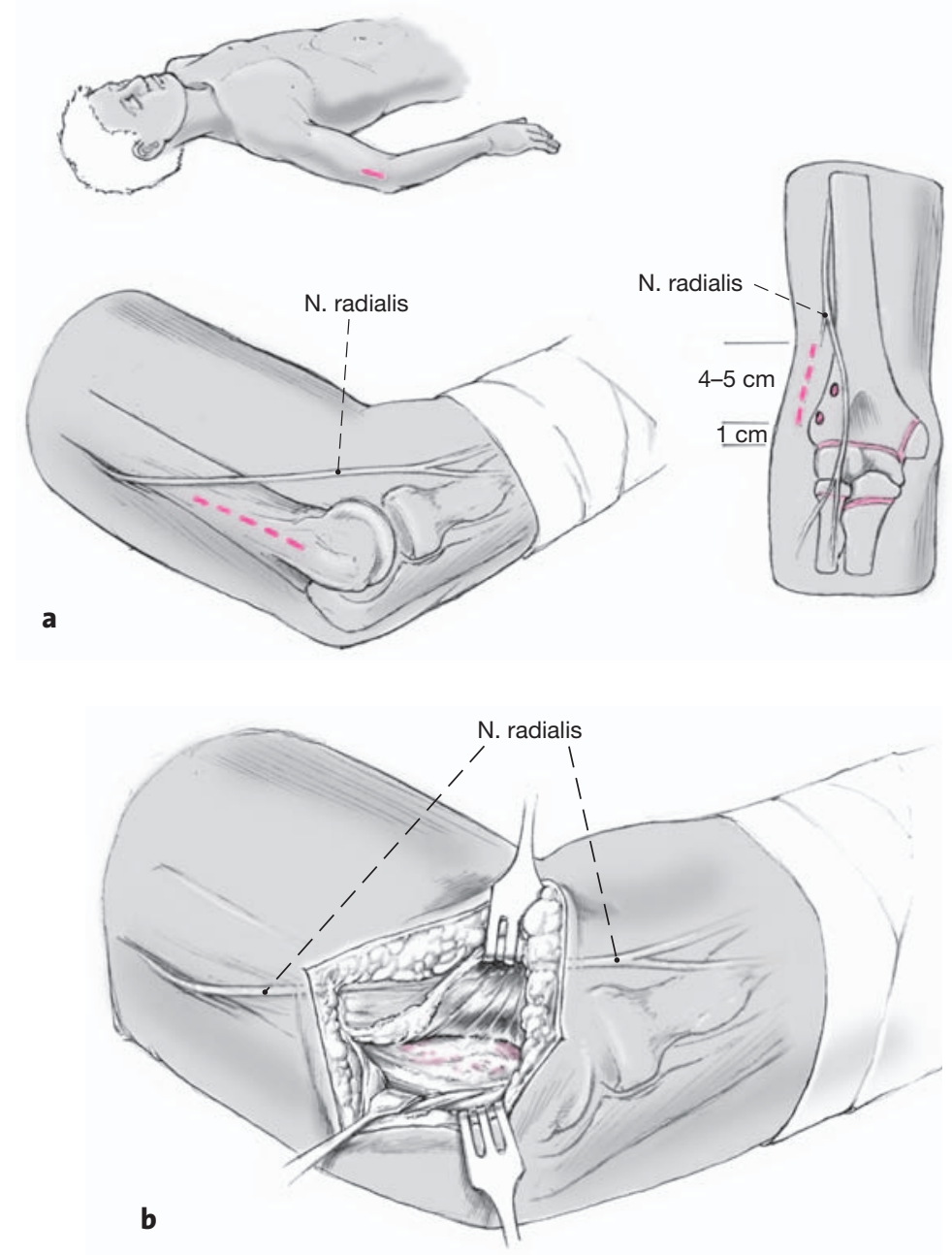

\section{Abbildung 6}

Hautinzision und Zugang; Abheben des Periosts.

Abheben des Periosts mit dem Raspatorium, um die lateroanteriore Region des distalen Humerus einsehen zu können. Die späteren Eintrittsstellen sind markiert. Wird zu weit proximal präpariert, ist der Nervus radialis in Gefahr.

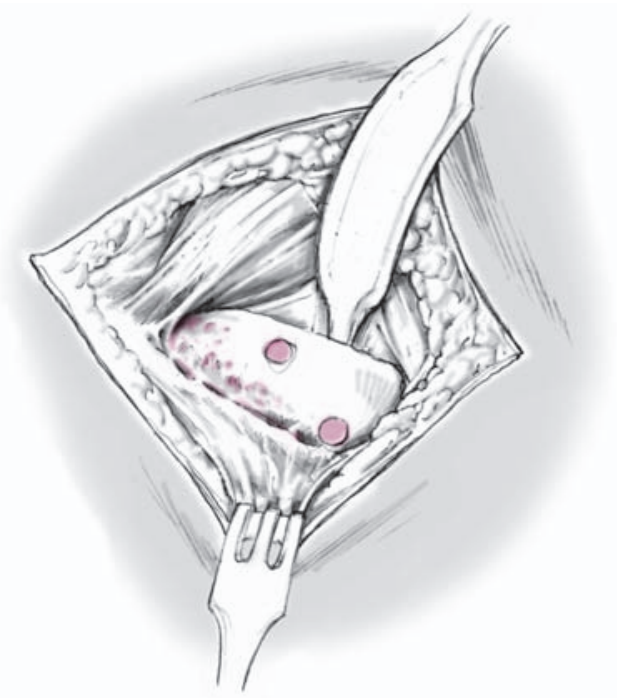




\section{Abbildung 7}

Eröffnung des Knochens.

Eröffnung des Humerus mit Pfriem oder Bohrer. Die Lage der beiden Eintrittsstellen ist dargestellt.

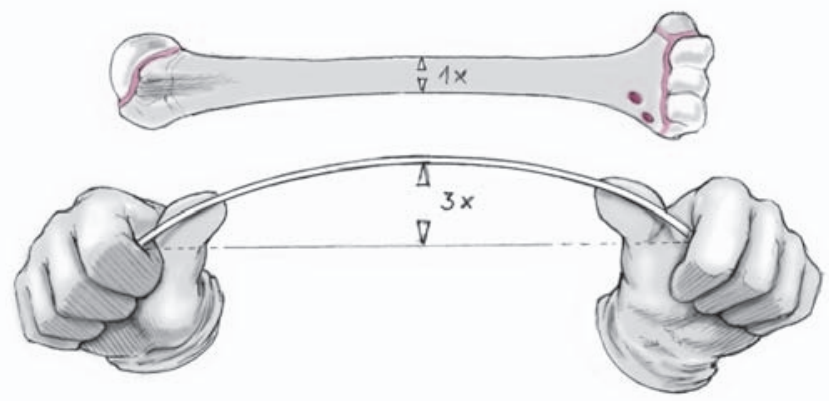

a

\section{Abbildungen $\mathbf{8 a}$ und $\mathbf{8 b}$}

Vorbiegen und Einführen des ersten Nagels.

C-förmiges Vorbiegen des ersten Nagels nur über der Knochenlänge respektive vorwiegend im proximalen Drittel etwa um den dreifachen Knochendurchmesser (a).

Das erste, distale Loch wird mit dem Pfriem oder Bohrer direkt auf der Crista $1 \mathrm{~cm}$ über der Fuge gebohrt (b, aus [1]). Unter Drehen des Pfriems oder bei laufendem Bohrer wird das Loch in einem Winkel von $50^{\circ}$ zum Schaft ausreichend aufgeweitet.
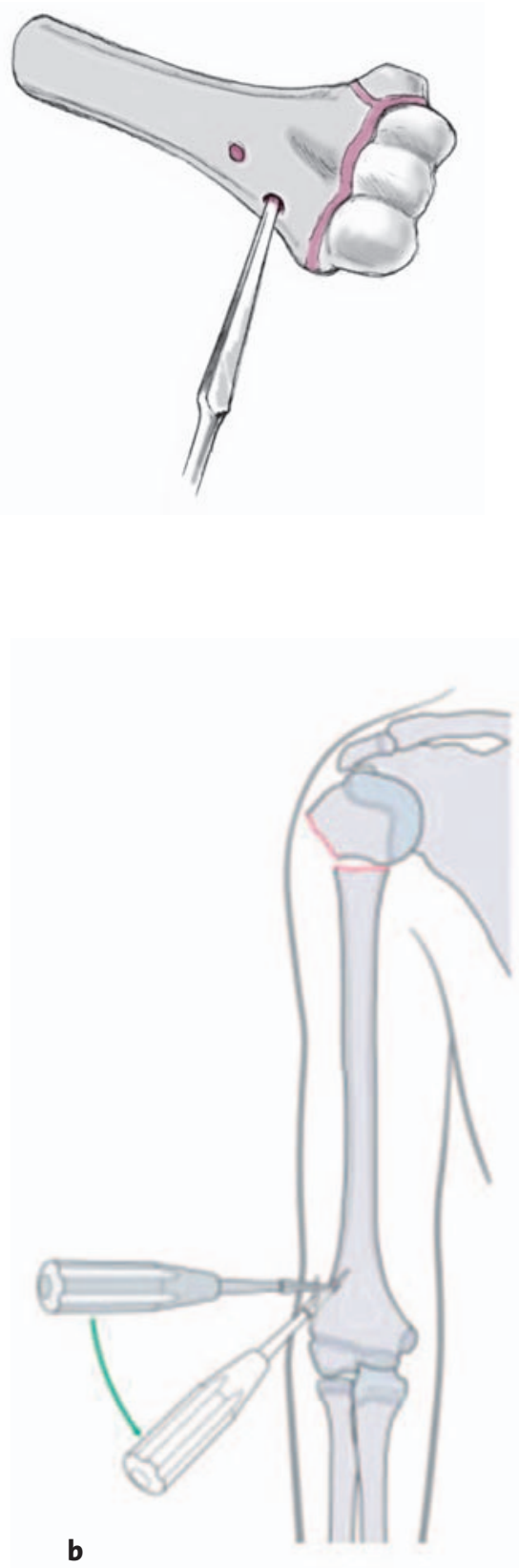

Einspannen des Nagels in das Einschlaginstrument und Einführen der Nagelspitze rechtwinklig zur Knochenoberfläche in das distale, erste Loch.

Danach Drehung des Nagels um $180^{\circ}$, damit sich die Nagelspitze parallel zur Knochenlängsachse ausrichtet.

Vortreiben des Nagels mit leichten Hammerschlägen auf den Inserter oder durch Hin- und Herbewegen des Nagels bis knapp vor die Fraktur. 


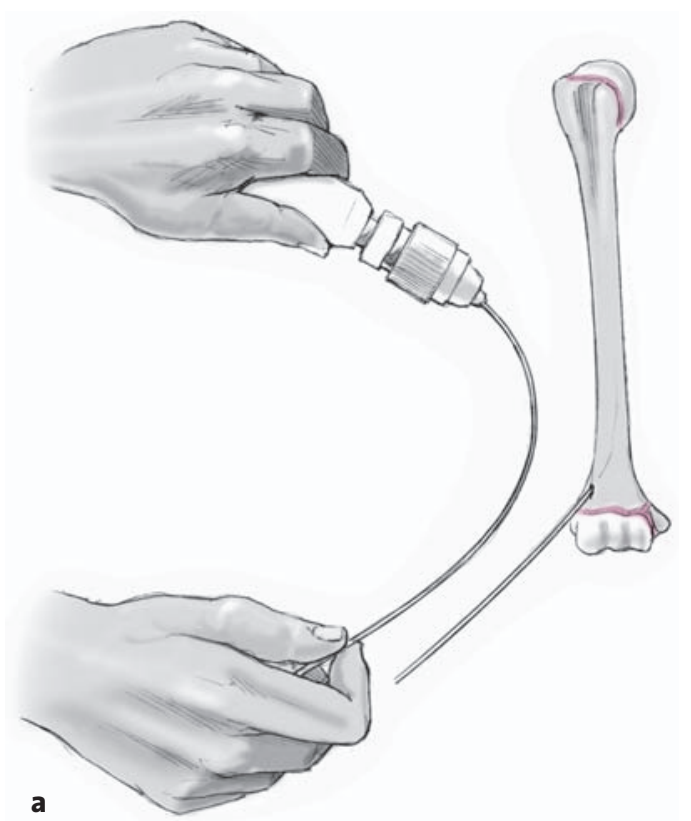

\section{Abbildungen 9a bis $9 c$}

Der zweite Nagels (a) wird nur im proximalen Drittel deutlich mehr als der erste vorgebogen; dies garantiert nach dem Drehen um $180^{\circ}$ eine gute innere Verspannung.

Das zweite Loch wird $1 \mathrm{~cm}$ proximal des ersten mehr auf dem ventralen Aspekt des Humerus gebohrt (b, aus [1]), und der zweite Nagel wird in gleicher Weise wie der erste eingeführt. Es ist darauf zu achten, ob der zweite Nagel ventral oder dorsal zum ersten zu liegen kommt, da dies die spätere Drehrichtung
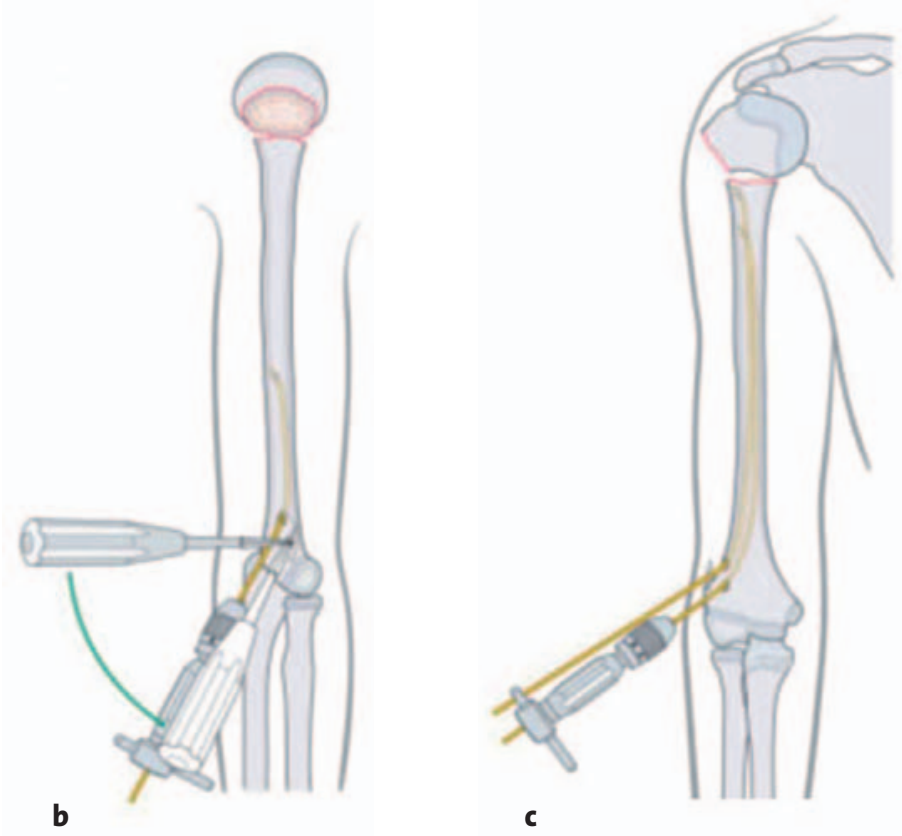

der Nägel beeinflusst! Vermeidung des Korkenzieherphänomens durch Berücksichtigung der jeweiligen Drehrichtung des Nagels.

Beide Nägel werden bis knapp unter die Fraktur geschoben (c, aus [1]).

Nachdem der zweite Nagel das proximale Drittel des Humerus erreicht hat, drehen wir ihn um $180^{\circ}$ im oder gegen den Uhrzeigersinn, je nach Lage des Nagels ventral oder dorsal des ersten. Die Nagelspitzen sind nun divergierend ausgerichtet. 


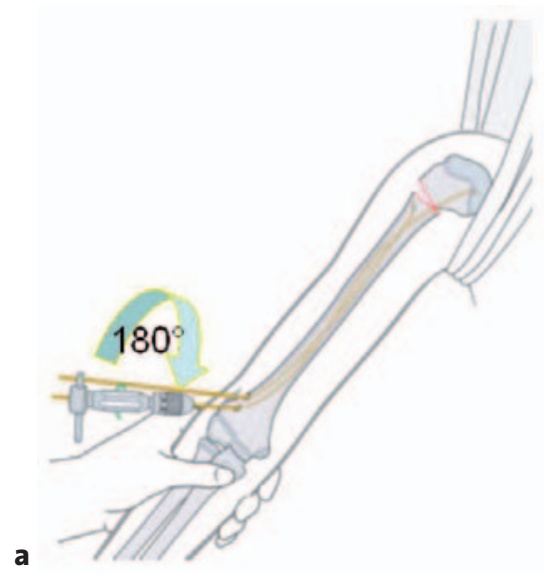

b

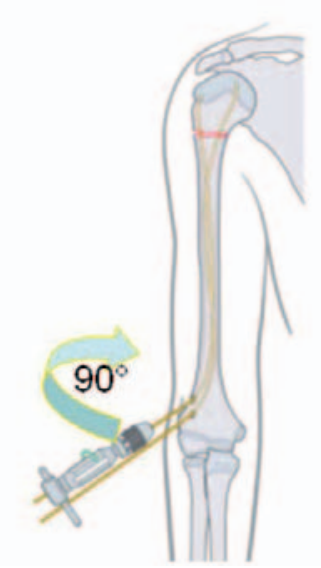

c

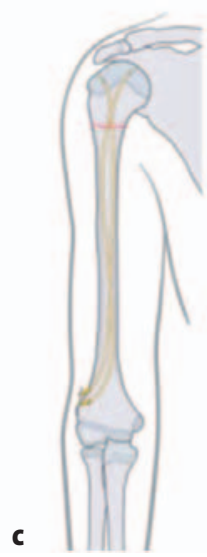

Anschließend wird der zweite Nagel über die Fraktur geschoben, und die definitive Reposition wird durch Drehung um $90^{\circ}$ erreicht (b, aus [1]).

Definitive Platzierung und Abschneiden der Nägel sowie korrektes, d.h divergierendes Ausrichten der Nagelspitzen (c, aus [1]).

Definitives Verankern und Absetzen der Nägel an der Eintrittsstelle.

Die verbleibende Länge ergibt sich aus der noch einzuschlagenden Strecke und einem geplanten Überstand von 5-6 mm. Vor der definitiven Verankerung der Nägel überprüfen wir die Rotation und Achse des Humerus nochmals klinisch und radiologisch. Mit dem abgeschrägten Einschlagbolzen definitive Verankerung der Nägel. Die proximale Epiphysenfuge darf dabei ohne Probleme einmalig mit dem Nagel perforiert werden. Wundverschluss.

Das abgelöste Periost wird mit Vicryl 2/o readaptiert, die Subkutanschicht mit Vicryl $3 / 0$ vernäht. Hautnaht mit Vicryl rapid 5/o als Einzelknopfnähte, fortlaufende oder intrakutane Nähte. Üblicher Wundverband gemäß Klinikschema. Elastische Binde, kein Gipsverband nötig! 


\section{Retrograde laterale und mediale Technik am Beispiel einer Humerusschaftfraktur}

\section{Abbildung 11}

Hautinzision.

Die radiale Inzision beginnt über dem Condylus radialis und zieht ca. 2,5-3 cm nach proximal. Die ulnare Inzision beginnt ca. $1 \mathrm{~cm}$ über dem Epicondylus ulnaris und zieht ebenfalls $2,5-3 \mathrm{~cm}$ nach proximal. Die Nageleintrittsstellen befinden sich bilateral am distalen Humerus. (Aus [1]).

\section{Abbildung 12}

Zugang zum Humerus.

Radial direktes Eingehen auf die laterale Crista humeri. Inzision des Periosts auf einer Länge von $3 \mathrm{~cm}$ und Abheben des Periosts mit dem Raspatorium, um die lateroanteriore Region des distale Humerus einsehen zu können. Wird zu weit proximal präpariert, ist der Nervus radialis in Gefahr. Ulnar muss die größere Distanz zum Knochen berücksichtigt werden. Stumpfes Eingehen auf den Humerus. Der Nervus ulnaris muss nicht unbedingt dargestellt werden, darf jedoch bei der Präparation keinesfalls verletzt werden.
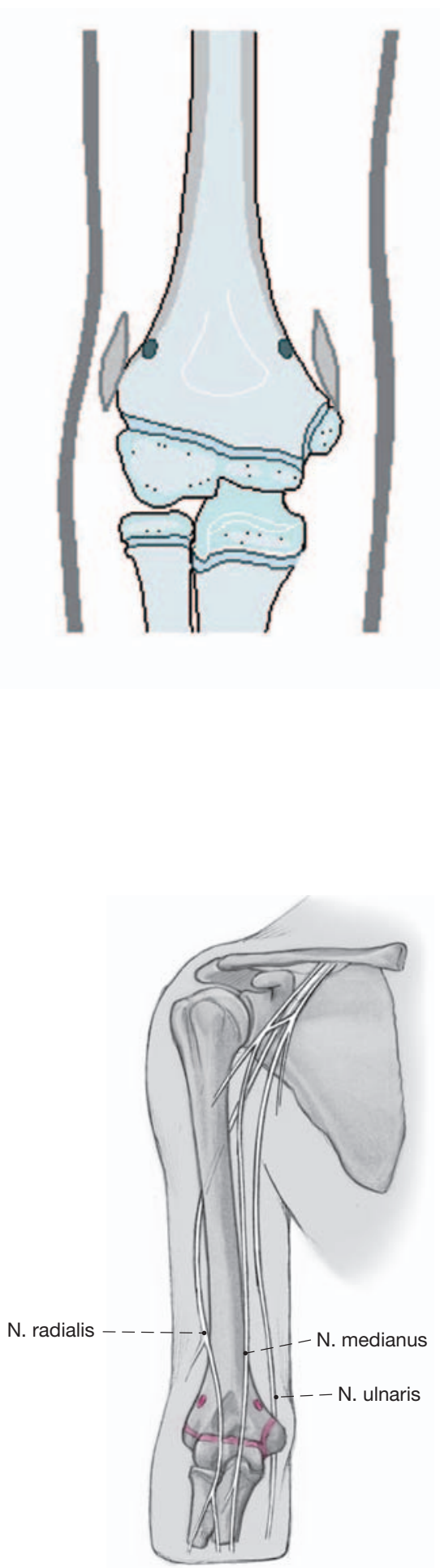

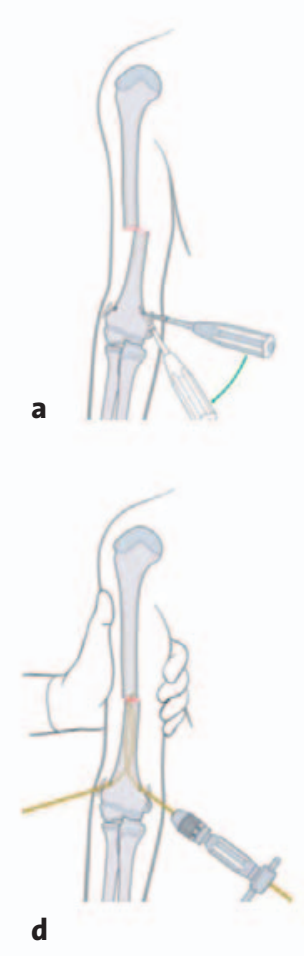

\section{Abbildungen 13a bis $13 f$}

Eröffnung des Knochens.

Radial wie ulnar Eröffnung des Knochens in der zuvor beschriebenen Technik (a). Man beachte, dass das Absenken des Pfriems ulnarseitig schwieriger ist, da der Epicondylus ulnaris vorsteht. Beim Rotieren des Pfriems oder Eröffnen des Knochens mit dem Bohrer muss eine Verletzung des Nervs unbedingt vermieden werden.

Einführen der Nägel.

Beide Nägel werden in gleicher Weise C-förmig über der Knochenlänge vorgebogen. Die Nägel sollten dieselbe Dicke aufweisen, da es sonst zu einer Fehlstellung durch die verschiedenen Nagelspannungen kommt. Einspannen des Nagels in das Einschlaginstrument und Einführen der Nagelspitze rechtwinklig zur Knochenoberfläche. Es hängt von der Morphologie der Fraktur ab, ob ulnar oder radial begonnen wird. Prinzipiell sollte mit dem Nagel begonnen werden, mit dem die Fraktur besser reponiert werden kann.

Die beiden Nägel werden in konventioneller Weise eingebracht und vorgeschoben (b, c). Dabei ist darauf achten, ob der zweite
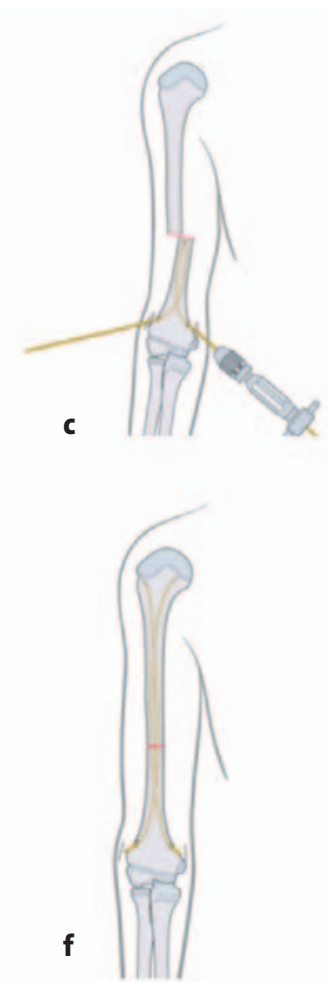

Nagel über oder unter dem ersten zu liegen kommt, da dies die spätere Drehrichtung beeinflusst. Vermeidung des Korkenzieherphänomens.

Indirekte Reposition von außen und mit Hilfe des besser zur Frakturebene liegenden Nagels. Kann dieser das proximale Fragment sicher fassen, wird er ca. 3-4 cm über die Frakturzone geschoben $(d, e)$.

Nachdem beide Nägel die Frakturzone überquert haben, wird die Fraktur korrekt ausgerichtet. Vor der definitiven Verankerung der Nägel prüfen wir Rotation und Achse nochmals klinisch und radiologisch.

Entsprechend der noch verbleibenden Einschlagtiefe Absetzen der beiden Nägel mit dem Schneidinstrument. Definitives Vorschlagen der Nägel mit dem Einschlagbolzen und gute Verankerung in der proximalen Metaphyse (f). (Aus [1]).

Wundverschluss.

Wie oben. 


\section{Anterograde proximale/radiale Technik für Frakturen des distalen Humerusdrittel und suprakondyläre Frakturen}

\section{Hautinzision.}

Inzision im Bereich des humeralen Ansatzes des Musculus deltoideus $2,5-3 \mathrm{~cm}$ nach proximal, um eine Hautverletzung beim Absenken des Pfriems um $45^{\circ}$ zu verhindern. Keine Inzision nach distal; dadurch käme man in den Bereich den Nervus radialis.

\section{Zugang zum Humerus.}

Stumpfes Präparieren bis auf den Knochen.

Einsetzen von zwei kleinen Weichteilhaken, um den Knochen sehen zu können. Die beiden Eintrittsstellen müssen wiederum in der Höhe mindestens $1,5 \mathrm{~cm}$ und in der Breite mindestens $1 \mathrm{~cm}$ versetzt sein (s. Abbildung 14), da es ansonsten zu einer Spaltung des Knochens kommen kann.
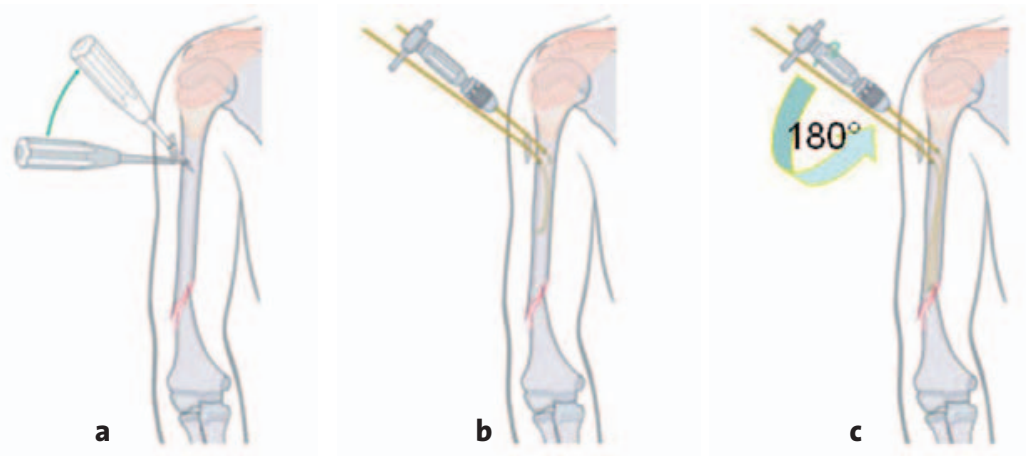

\section{Abbildungen 14a bis $14 \mathrm{~g}$ Eröffnung des Knochens.}

Zunächst wird das proximale Loch mit dem Pfriem oder dem entsprechenden Bohrer eröffnet; es sollte genau lateral liegen (a). Das zweite Loch wird in gleicher Weise eröffnet, nachdem der erste Nagel bereits eingeführt ist. Dies erleichtert die Identifikation der Eintrittsstelle.

Einführen der Nägel.

Die Technik entspricht der monolateralen, radialen Technik (b): C-förmiges Vorbiegen des ersten Nagels, einerseits nur über der Knochenlänge, andererseits vorwiegend im proximalen Drittel etwa um den dreifachen Knochendurchmesser. Einspannen des Nagels in das Einschlaginstrument und Einführen der Nagelspitze rechtwinklig zur Knochenoberfläche in das distale, erste Loch. Danach Drehung des Nagels um $180^{\circ}$, damit sich die Nagelspitze parallel zur Knochenlängsachse ausrichtet. Vorschieben des Nagels mit leichten Hammerschlägen auf den Inserter oder unter Hin- und Herbewegung des Nagels bis knapp vor die Fraktur.

Starkes Vorbiegen des zweiten Nagels nur im proximalen Drittel. Der zweite Nagel wird wie der erste in das zweite, weiter distal gelegene Loch eingeführt und ebenfalls knapp bis vor die Fraktur geschoben. Drehung dieses nur im proximalen Anteil stark C-förmig vorgebogenen Nagels um $180^{\circ}$ (c). Dabei ist darauf zu achten, ob der zweite Nagel über oder unter dem ersten
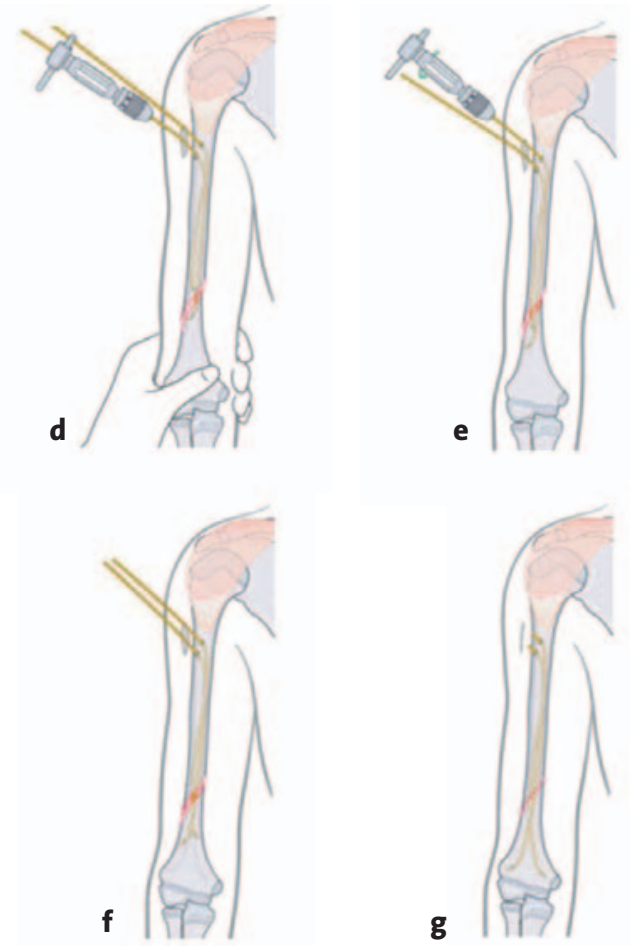

zu liegen kommt, da dies die spätere Drehrichtung beeinflusst. Vermeidung des Korkenzieherphänomens.

Mit dem ersten, C-förmig vorgebogenen Nagel Vordringen bis zur Fraktur und indirekte Reposition der Fraktur unter gleichzeitigem Zug von außen (d). Überqueren der Fraktur und Vorschieben in das distale Fragment. Gleiches Vorgehen mit dem zweiten Nagel (e). Vor der definitiven Verankerung der Nägel sind die Rotation und Achse nochmals klinisch und radiologisch zu überprüfen.

Entsprechend der noch verbleibenden Einschlagtiefe Absetzen der beiden Nägel mit dem Schneidinstrument (f). Proximal sollten die Nägel länger belassen werden, damit sie nicht unter der Muskulatur zu liegen kommen, sondern bis knapp subfaszial reichen. Dies erleichtert auch ihre Entfernung. Definitives Vorschlagen der Nägel mit dem Einschlagbolzen und gute Verankerung in der distalen Metaphyse (g). (Aus [1]).

Gipsverbandfreie Nachbehandlung. 


\title{
Besonderheiten (Abbildung 15)
}

\begin{abstract}
Abbildung 15
Suprakondyläre Humerusfraktur.

Analoges Vorgehen. Die beiden Nägel müssen dann jedoch bis in beide distalen metaphysären Pfeiler geschoben werden. Die Fraktur muss perfekt reponiert sein, ansonsten finden die beiden Nägel den Weg nicht in den radialen und ulnaren Pfeiler. Die Nägel werden bis an die distale Fuge, bei kurzem Fragment über die Fuge hinaus eingeschlagen. In diesen Fällen ist es ratsam, die Nagelspitzen leicht schräg anzuschneiden, damit sie spitzer werden und sich leichter in die sehr harte distale Metaphyse einbringen lassen.

Eine einwandfreie Reposition ist bei der ESIN-Technik unerlässlich. Falls Repositionsprobleme bestehen, muss die Fraktur über eine kurze radiale Inzision dargestellt und reponiert werden. Die Nägel können dann unter Sicht direkt in das distale Fragment geführt werden. Dabei sollte zuerst der ulnare, dann der radiale Nagel vorgeschoben werden. (Aus $[1,6]$ ).
\end{abstract}
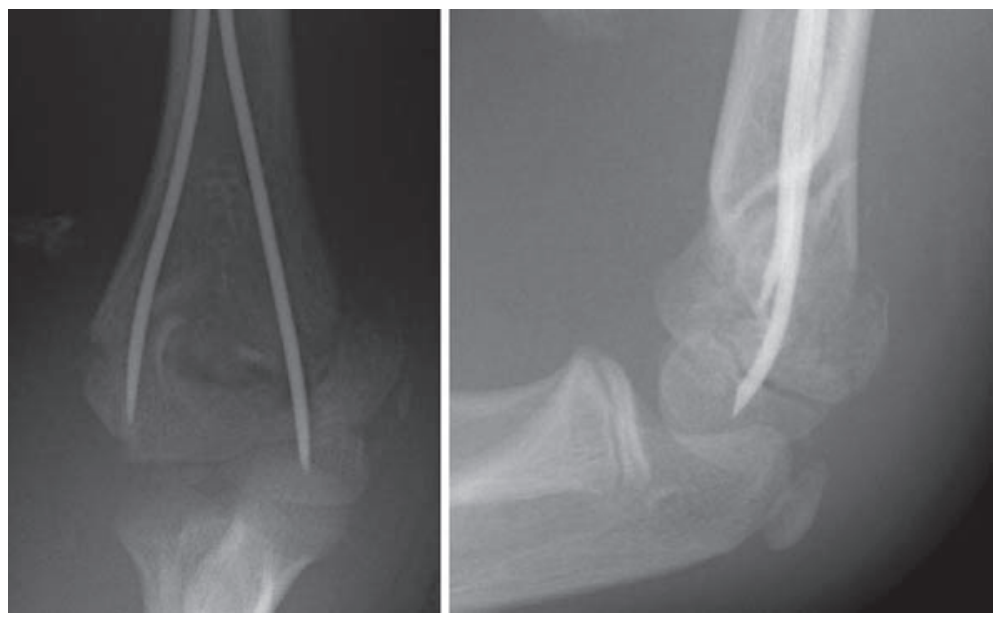

\section{Postoperative Behandlung}

- Postoperatives Röntgen mit langer Platte in zwei Ebenen einschließlich des Schulter- und Ellbogengelenks.

- Kein Gipsverband.

- Unbelastete, freie Bewegung erlaubt.

- Hospitalisierung 1-2 Tage, besser tagesambulant.

- Erste klinische und radiologische Kontrolle nach 4-5 Wochen je nach Alter.

- Sportfreigabe je nach knöcherner Heilung.

- Hausarztkontrollen sind bei Verwendung resorbierbarer Fäden nicht notwendig.

- Abschließende Röntgenkontrolle vor Implantatentfernung.

- Implantatentfernung nach 4-6 Monaten.

\section{Fehler, Gefahren, Komplikationen}

- Aufwickelung oder Perforation des Nervus radialis beim Versuch, das proximale Fragment bei Schaftfrakturen zu fassen: Kleine Inzision über dem Nerv, um ihn sicher aus der Frakturregion zu halten. Auch bei fehlender oder unmöglicher Reposition sollten über eine kleine Inzision die Frakturenden dargestellt und die Nägel unter Sicht vorgeschoben werden.
- Hautirritationen bei zu lang abgesetzten Nägeln: Gute Pflege der Weichteile und Abwarten bis zur Implantatentfernung.

- Postoperative Läsion des Nervus radialis: Revision des Nervs, da der Nagel diesen evtl. in der Frakturregion verletzt hat.

- Rotationsfehler bei Frakturen am proximalen Humerus und Schaft: Keine besonderen Maßnahmen erforderlich, da sie durch das Schultergelenk gut kompensiert werden. Bei suprakondylären Frakturen kommen Rotationsfehler nicht vor, da die Nägel in den Pfeilern eine korrekte Stellung garantieren [7].

- Fehlstellungen durch ungleich hohe, also asymmetrische Eintrittspunkte bei der bilateralen Nagelung und dadurch unterschiedlich große innere Spannung (Abbildung 16a).

- Verringerte Stabilität der Nagelung und Reduktion auf eine simple Schienung durch das sog. Korkenzieherphänomen (Abbildung 16b). Durch die Verdrehung der Nägel untereinander verlieren diese den Kontakt zur inneren Kortikalis, die Stabilität geht dabei verloren. 


\section{Abbildungen 16a und 16b}

a) Fehlstellungen durch ungleich hohe, also asymmetrische Eintrittspunkte.

b) Korkenzieherphänomen. (Aus [1]).
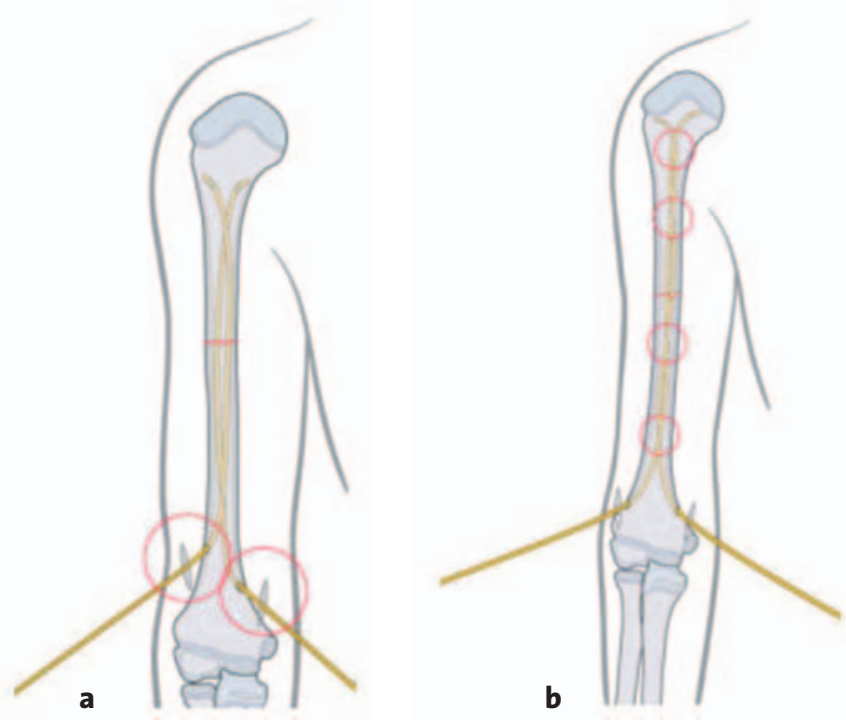

\section{Ergebnisse}

Aufgrund der enormen Kompensationsfähigkeit der Schulter sowie der hohen Remodellierungspotenz besonders des proximalen Humerus sind die Resultate durchweg gut bis sehr gut.

Lediglich chirurgisch-technische Probleme können zu einem schlechten Ergebnis führen [1, 4, 6].

Vorübergehende Nervenirritationen gehen spontan zurück und bedürfen keiner Revision. Ob heute auf eine Implantatentfernung verzichtet werden sollte, ist umstritten; meist stehen ökonomische Überlegungen dahinter.

\section{Literatur}

1. Dietz HG, Schmittenbecher PP, Slongo TF, et al. AO manual of fracture management. Elastic stable intramedullary nailing (ESIN) in children. Stuttgart: Thieme, 2006.

2. Gautier E, Slongo T, Jakob RP. Die Behandlung der subkapitalen Humerusfraktur mit dem Prevot-Nagel. Z Unfallchir Versicherungsmed 1992; $85: 145-55$

3. Havranek P, PesI T. [Use of the elastic stable intramedullary nailing technique in non-typical paediatric fractures.] Acta Chir Orthop Traumatol Cech 2002;69:73-8.

4. Lascombes P, Haumont T, Journeau P. Use and abuse of flexible intramedullary nailing in children and adolescents.J Pediatr Orthop 2006;26: $827-34$.
5. Metaizeau JP. Ostéosynthèse chez l'enfant. Montpellier: Sauramps Medical, 1988

6. Schmittenbecher PP, Blum J, David S, et al. Die Behandlung von Humerusschaftfrakturen und subkapitalen Humerusfrakturen im Kindesalter. Unfallchirurg 2004;107:8-14.

7. Schmittenbecher PP, Dietz HG, Linhart WE, et al. Complications and problems in intramedullary nailing of children's fractures. Eur J Trauma 2000;26:287-93.

8. Slongo T, Audigé L, Schlickewei W, et al. Development and validation of the $\mathrm{AO}$ paediatric comprehensive classification of long bone fractures by the Paediatric Expert Group of the AO Foundation in Collaboration with AO Clinical Investigation and Documentation. J Pediatr Orthop 2006;26:43-9.

\section{Korrespondenzanschrift}

Dr. Theddy Slongo

Leitender Arzt für Kindertraumatologie und Kinderorthopädie Abteilung für Pädiatrische Chirurgie Universitätskinderklinik Inselspital

3010 Bern

Schweiz

Telefon (+41/31) 632-9293, Fax -9292

E-Mail: theddy.slongo@insel.ch 